\title{
Prognostic factors for mortality with febrile neutropenia in hospitalized patients
}

\author{
Nattamol Hosiriluck MD, Saranapoom Klomjit MD, Supannee Rassameehiran MD, \\ Grerk Sutamtewagul MD, Lukman Tijani MD, Saba Radhi MD
}

\begin{abstract}
Background: Febrile neutropenia is a life-threatening complication of cancer treatment that results in hospitalization and delays cancer therapy. The aim of this study is to identify prognostic factors of patients admitted with febrile neutropenia.

Methods: A retrospective study via chart review without direct patient contact was conducted. All adults above 18 years with the diagnosis of febrile neutropenia hospitalized in University Medical Center in Lubbock, Texas, between January 2010 and December 2013 were reviewed. The data were analyzed with IBM SPSS statistics.

Results: One hundred twenty-seven patients were included in this study. The hospital mortality rate was $17.3 \%$, and the 30 -day mortality rate was $20.5 \%$. The median length of stay (LOS) was eight days. Hematologic malignancies accounted for $66.1 \%$ of the patients. On multivariate analysis, the in-hospital mortality rate was associated with fever duration (adjusted odds ratio [OR] 7.19; 95\% Cl 1.06-50.00; $p<0.04$ ), abnormal liver function tests (adjusted OR 63.72; 95\% Cl 5.95-682.07; $p<0.001$ ), ICU admission (OR 45.78; 95\% Cl 4.97-420.99; $p<0.001$ ) and positive culture (adjusted OR 12.71; 95\% Cl 1.14-142; $p$ <.039). The independent risk factors for a 30-day mortality rate were abnormal liver enzymes (OR 48.38; 95\% CI 5.27-444.29; $p<0.001$ ), ICU admission (OR 63.66; $95 \% \mathrm{Cl} 5.96-680.30 ; p<0.001)$ and fever duration more than four days (OR 8.26; 95\% Cl 1.11-62.50; $p=0.039)$. The data indicated that diagnosis of hematologic malignancies (OR 4.06; 95\% Cl 1.34-12.31 p=0.013), fever duration (adjusted OR 6.29; 95\% $\mathrm{Cl} 1.81-21.92 ; \mathrm{p} \mathrm{0.004)}$ and neutropenic duration more than five days (OR 3.68; $95 \% \mathrm{Cl}$ 1.44-9.40 $p=0.007$ ) were associated with LOS more than eight days.

Conclusions: Febrile neutropenia in hospitalized patients results in a significant mortality rate. Factors associated with increased mortality include ICU admission and abnormal liver enzyme tests.
\end{abstract}

Key words: Febrile neutropenia, cancer, ICU admission, liver enzyme tests, mortality, and length of stay

Corresponding author: Nattamol Hosiriluck, MD Contact Information: Nattamol.hosiriluck@ ttuhsc.edu.

DOI: $10.12746 /$ swrccc2015.0309.112

\section{INTRODUCTION}

Febrile neutropenia is a life threatening complication of cancer treatment that results in hospitaliza- 
tion and the need for broad spectrum antibiotics. It can affect outcomes during cancer treatment through delays in the treatment cycle, dose reductions, or discontinuation of chemotherapy. Its consequences depend on the severity and duration of the neutropenic phase and vary greatly among patient groups. Febrile neutropenia accounts for approximately $40 \%$ to $50 \%$ of the total cost of hospitalization in cancer care and results in mortality rates between $3 \%$ and $20 \%$, depending on risk factors. ${ }^{1,2}$ Risk stratification could influence the medical management in febrile neutropenic patients, and high risk patients might benefit from a more intensive medical approach. The current National Comprehensive Cancer Network (NCCN) guideline recommends the use of the Multinational Association for Supportive Care in Cancer (MASCC) Risk Index (Table 1) as a risk stratification tool to identify low risk febrile neutropenic patients. ${ }^{3}$ However, this index stratifies patients into only outpatient and inpatient management groups. There is no risk scoring system available for febrile neutropenic patients who require hospitalization, but there have been studies on prognostic factors to improve risk assessment of these patients based on clinical and laboratory indicators. Some of these factors include the duration and severity of neutropenia, the underlying cancer, disease status, underlying comorbidities, and types of infection. ${ }^{4,5}$ In our study we wanted to identify prognostic factors for febrile neutropenia in hospitalized patients.

Table 1 The Multinational Association for Supportive Care in Cancer (MASCC) Risk Index

\begin{tabular}{|lc|}
\hline \multicolumn{1}{|c}{ Characteristic } & Weight \\
Burden of illness: no or mild symptoms & 5 \\
No hypotension & 5 \\
No chronic obstructive pulmonary disease & 4 \\
Solid tumor or no previous fungal infection & 4 \\
No dehydration & 3 \\
Burden of illness: moderate symptoms & 3 \\
Outpatient status & 3 \\
Age $<60$ years & 2 \\
\hline
\end{tabular}

Points attributed to the variable "burden of illness" are not cumulative. The maximum theoretical score is therefore 26 . $\geq 21$ : low risk patients (positive predictive value $91 \%$, specificity $68 \%$, sensitivity $71 \%$ )

\section{Material and Methods}

\section{Patient population}

A retrospective study via chart review without direct patient contact was conducted. All patients aged greater than 18 years old with a diagnosis of cancer and ongoing chemotherapy treatment with fever (temperature equivalent to $\geq 38.0^{\circ} \mathrm{C}$ ) and neutropenia (absolute neutrophil count less than $500 / \mathrm{mcL}$ and/or a predicted decline to less than $500 / \mathrm{mcL}$ over next 48 hours) hospitalized in University Medical Center in Lubbock, Texas, from January 1, 2010, to December 31,2013 , were included in the study.

Inclusion criteria were based on the ICD 9 288.00 code for the diagnosis of febrile neutropenia. All data were reviewed and checked for accuracy. Some patients were admitted initially for infection or chemotherapy and did not have febrile neutropenia at the time of admission but later developed it. This group of patients was also included as a secondary diagnosis of febrile neutropenia with the onset of fever occurring as inpatient. Some patients developed multiple episodes of febrile neutropenia, but only the first one was considered in this study. Exclusion criteria included patients who were on palliative care measures and who did not receive or denied treatment. Febrile neutropenia from all causes other than chemotherapy (e.g., primary immunodeficiency or liver disease) was also excluded from the study. All patients' data were deidentified and included the patients' demographics (ages, gender, types of cancer, and comorbidities) and clinical parameters, including complete blood count with absolute neutrophil count (ANC), complete metabolic panel, and culture at diagnosis of febrile neutropenia. All patients' hospital courses were reviewed to determine length of stay (LOS) and discharge outcome. This study was approved by the Institutional Board Review for the protection of human subjects of the Texas Tech Health Sciences Center, Lubbock/Odessa, Texas.

\section{Outcome meAsures}

The primary outcome was inpatient mortality. Factors associated with 30-day mortality rates and 
LOS were also assessed.

\section{Statistical analysis}

Descriptive analysis was used to describe patient characteristics. Univariate and multi-variable logistic regression models were used to evaluate the association between clinical risk factors and patient mortality and LOS. A $p$ value less than 0.05 was considered statistically significant. The data were analyzed with the IBM SPSS software (IBM; version).

\section{RESULTS}

The first episodes of febrile neutropenia in 127 patients were included in the study. The cohort included 80 men $(63 \%)$ and 47 women $(37 \%)$ with a mean age of $55.7 \pm 16.5$ (range 19-84). Most patients were more than 55 years. The ethnic/race distribution included 81Caucasians (63.8\%), 43 Hispanics (33.9\%), and three African-Americans (2.4\%). Twelve patients $(9.4 \%)$ had metastatic disease at the time of febrile neutropenia diagnosis. Eighty-five patients (66.9\%) had one or more comorbidities. The most common comorbidities were essential benign hypertension in 59 patients $(46.5 \%)$, diabetes in 23 patients $(18.1 \%)$, and chronic kidney disease in 16 patients (12.6\%). Thirty-six patients (28.3\%) were admitted to or transferred to the intensive care unit (ICU) during hospitalization.

The mean fever duration was $3.5 \pm 3.75$ days (median: two days, interquartile range [IQR]:1-4 days; range 1-26 days). The mean absolute neutrophil count at the onset of fever was $629.32 / \mathrm{mcl}$ with mean lowest count at $9.49 / \mathrm{mcl}$. The number of days from onset of fever to lowest ANC count was $2 \pm 1.8$ days. Colony stimulating factor was given to 96 patients $(75.6 \%)$. The neutropenic period ranged from 1-22 days with a median of five days. Every patient was on antibiotics at the time that fever developed. The sources of infection were unknown in 46 cases $(36.2 \%)$, the lung in 21 cases $(16 \%)$, the gastrointestinal tract in 21 cases $(16 \%)$, the urinary tract in 16 cases (12.6\%), and primary bacteremia in 17 cases $(13.4 \%)$. Seventy-one cases $(55.9 \%)$ had positive cultures, including 38 Gram positive cultures (29.9\%), 32 Gram negative cultures (25.2\%), and one fungal culture $(0.8 \%)$.

The hospital mortality rate was $17.3 \%$, and the 30 -day mortality rate was $20.5 \%$. The LOS ranged from 2-73 days with a median of eight days (IQR 5-14 days). Seventy-one patients (55.9\%) had a LOS more than eight days. A MASCC score less than 21 occurred in 22 of 26 patients (84.62\%) in the 30-day mortality rate group, but this was not statistically different from the score in 30-day survivors.

Univariate analysis showed that abnormal liver enzymes, ICU admission, fever duration more than four days, and positive cultures were significantly associated with death (Tables 3 and 4). The type of malignancy and neutropenic duration were associated with prolonged LOS (> 8 days) (Table 5). On multivariate analysis, the in-hospital mortality rate was associated with fever duration (adjusted odds ratio [OR] 7.19; 95\% Cl 1.06-50.00; $p<0.04$ ), abnormal liver enzymes (adjusted OR 63.72; 95\% Cl 5.95-682.07; $\mathrm{p}<0.001$ ), ICU admission (OR 45.78; 95\% Cl 4.97420.99; $p<0.001$ ) and positive culture (adjusted OR $12.71 ; 95 \% \mathrm{Cl} 1.14-142 ; p<0.039)$. The independent risk factors for a 30-day mortality rate were abnormal liver enzymes (OR 48.38; 95\% Cl 5.27-444.29; $\mathrm{p}<0.001$ ), ICU admission (OR 63.66; 95\% Cl 5.96$680.30 ; p<0.001)$ and fever duration more than four days (OR 8.26; 95\% Cl 1.11-62.50; $p=0.039$ ). The data indicated that diagnosis of hematologic malignancies (OR 4.06; 95\% Cl 1.34-12.31 p=0.013), fever duration (adjusted OR 6.29; 95\% Cl 1.81-21.92; $p=0.004)$ and neutropenic duration more than five days (OR 3.68; 95\% Cl 1.44-9.40 $p=0.007$ ) were associated with LOS more than eight days (Tables 3, 4 and 5).

\section{Discussion}

Febrile neutropenia is a major complication from cancer treatment that carries a high mortality rate. Lyman et al 6 studied a large cohort of patients with cancer and found that patients who developed febrile neutropenia had a higher overall and early mortal- 
Table 2 Characteristics of patients in study (total $\mathrm{N}=127$ )

\begin{tabular}{|c|c|c|c|}
\hline & & $\mathbf{N}$ & Percent \\
\hline \multirow[t]{2}{*}{ Gender } & Male & 80 & 63 \\
\hline & Female & 47 & 37 \\
\hline \multirow[t]{3}{*}{ Race } & White & 81 & 63.8 \\
\hline & Hispanic & 43 & 33.9 \\
\hline & African-American & 3 & 2.4 \\
\hline \multirow[t]{8}{*}{ Diagnosis } & Leukemia & 58 & 45.7 \\
\hline & Lymphoma & 26 & 20.5 \\
\hline & GI malignancy & 10 & 7.9 \\
\hline & Lung cancer & 6 & 4.7 \\
\hline & Soft tissue & 3 & 2.4 \\
\hline & Head and Neck & 3 & 2.4 \\
\hline & breast cancer & 6 & 4.7 \\
\hline & Other & 15 & 11.8 \\
\hline \multirow[t]{7}{*}{ Comorbidities } & Diabetes Mellitus & 23 & 18.1 \\
\hline & Hypertension & 59 & 46.5 \\
\hline & Heart failure & 10 & 7.9 \\
\hline & Chronic kidney disease & 16 & 12.6 \\
\hline & Deep vein thrombosis & 6 & 4.7 \\
\hline & COPD/asthma & 11 & 8.6 \\
\hline & Coronary artery disease & 11 & 8.6 \\
\hline \multirow{2}{*}{ MASCC score } & $<21$ & 92 & 72.4 \\
\hline & $>21$ & 35 & 27.6 \\
\hline ICU admission & & 36 & 28.3 \\
\hline 30 day mortality & & 26 & 20.5 \\
\hline In hospital mortality & & 22 & 18.9 \\
\hline \multirow[t]{3}{*}{ Discharge destination } & Home & 89 & 70.1 \\
\hline & Nursing facility & 5 & 3.9 \\
\hline & Hospice & 9 & 7.1 \\
\hline CSF & & 96 & 75.6 \\
\hline
\end{tabular}


Table 2 Characteristics of patients in study (total $\mathrm{N}=127$ ) (Continued)

\begin{tabular}{|llcc|}
\hline \multirow{2}{*}{ Source of infection } & & $\mathrm{N}$ & Percent \\
& Pneumonia & 21 & \\
& UTI & 16 & 16.5 \\
& Primary bacteremia & 17 & 12.6 \\
& gastrointestinal & 21 & 13.4 \\
& Skin/soft tissue & 2 & 16.5 \\
& Other & 4 & 1.6 \\
Culture & Positive & 71 & 3.1 \\
Gram & Negative & 56 & 55.9 \\
& Positive & 38 & 44.1 \\
Readmission in 30 days & Negative & 33 & 29.9 \\
\hline
\end{tabular}

Table 3 Prognostic factors associated with in-hospital mortality in febrile neutropenia patients

\begin{tabular}{|c|c|c|c|c|c|}
\hline Variable & $\begin{array}{l}\text { In hospital } \\
\text { Expired }(n=22)\end{array}$ & $\begin{array}{l}\text { Crude OR } \\
\text { (Cl) }\end{array}$ & P-Value & $\begin{array}{l}\text { Adjusted OR } \\
\text { (CI) }\end{array}$ & P-Value \\
\hline \multicolumn{6}{|l|}{ Age (years) } \\
\hline$\leq 60$ & $14(20.6 \%)$ & 1.65 & 0.3 & 1.86 & 0.57 \\
\hline$>60$ & $8(13.6 \%)$ & $(0.64-4.27)$ & & $(0.22-16.13)$ & \\
\hline \multicolumn{6}{|l|}{ Gender } \\
\hline Male & $15(18.8 \%)$ & 1.32 & 0.58 & 3.61 & 0.18 \\
\hline Female & $7(14.9 \%)$ & $(0.50-3.51)$ & & $(0.55-23.55)$ & \\
\hline \multicolumn{6}{|l|}{ Race } \\
\hline White & $15(18.5 \%)$ & 1.1 & 0.83 & 1.61 & 0.522 \\
\hline Hispanic & $6(14 \%)$ & $(0.46-2.64)$ & & $(0.37-6.95)$ & \\
\hline African-American & $1(0.5 \%)$ & & & & \\
\hline \multicolumn{6}{|l|}{ Type } \\
\hline $\begin{array}{l}\text { Hematologic } \\
\text { Malignancies }\end{array}$ & $16(19 \%)$ & 1.74 & 0.32 & 1.16 & 0.334 \\
\hline Solid tumor & $5(11.9 \%)$ & $(0.59-5.13)$ & & $(0.13-10.41)$ & \\
\hline
\end{tabular}


Table 3 Prognostic factors associated with in-hospital mortality in febrile neutropenia patients (Continued)

\begin{tabular}{|c|c|c|c|c|c|}
\hline Variable & $\begin{array}{l}\text { In hospital } \\
\text { Expired }(n=22)\end{array}$ & $\begin{array}{l}\text { Crude OR } \\
\text { (CI) }\end{array}$ & P-Value & $\begin{array}{l}\text { Adjusted OR } \\
\text { (CI) }\end{array}$ & P-Value \\
\hline $\begin{array}{l}\text { Comorbidities } \\
\text { Yes } \\
\text { No }\end{array}$ & $\begin{array}{l}12(14.1 \%) \\
10(23.8 \%)\end{array}$ & $\begin{array}{l}0.526 \\
(0.21-1.34)\end{array}$ & 0.18 & $\begin{array}{l}1.01 \\
(0.16-6.40)\end{array}$ & 0.717 \\
\hline $\begin{array}{l}\text { Fever duration } \\
>4 \text { day } \\
<4 \text { day }\end{array}$ & $\begin{array}{l}12(38.7 \%) \\
10(10.4 \%)\end{array}$ & $\begin{array}{l}5.43 \\
(2.05-14.40)\end{array}$ & $<0.001$ & $\begin{array}{l}7.19 \\
(1.06-50.00)\end{array}$ & $0.04^{* *}$ \\
\hline $\begin{array}{l}\text { Liver enzymes } \\
\text { Abnormal } \\
\text { Normal }\end{array}$ & $\begin{array}{c}15(46.9 \%) \\
7(7.4 \%)\end{array}$ & $\begin{array}{l}11.43 \\
(3.27-39.87)\end{array}$ & $<0.001$ & $\begin{array}{l}63.72 \\
(5.95-682.07)\end{array}$ & $<0.001^{* *}$ \\
\hline $\begin{array}{l}\text { Albumin level } \\
\text { Normal (> 3g/dL) } \\
\text { Low }\end{array}$ & $\begin{array}{r}6(20.7 \%) \\
16(16.3 \%)\end{array}$ & $\begin{array}{l}1.34 \\
(0.47-3.81)\end{array}$ & 0.58 & $\begin{array}{l}3.62 \\
(0.54-24.39)\end{array}$ & 0.38 \\
\hline $\begin{array}{l}\text { GFR } \\
<60 \\
>60\end{array}$ & $\begin{array}{r}9(24.3 \%) \\
13(14.8 \%)\end{array}$ & $\begin{array}{l}1.85 \\
(0.71-4.82)\end{array}$ & 0.21 & $\begin{array}{l}1.41 \\
(0.26-7.68)\end{array}$ & 0.69 \\
\hline $\begin{array}{l}\text { MASCC } \\
\text { High risk } \\
\text { Low risk }\end{array}$ & $\begin{array}{c}18(19.6 \%) \\
4(11.4 \%)\end{array}$ & $\begin{array}{l}1.89 \\
(0.59-6.20)\end{array}$ & 0.29 & $\begin{array}{l}5.39 \\
(0.51-56.63)\end{array}$ & 0.16 \\
\hline $\begin{array}{l}\text { Neutropenic perio } \\
>5 \text { day } \\
\leq 5 \text { day }\end{array}$ & $\begin{array}{r}14(20.3 \%) \\
8(14.3 \%)\end{array}$ & $\begin{array}{l}1.53 \\
(0.59-3.95)\end{array}$ & 0.38 & $\begin{array}{l}1.16 \\
(0.24-6.25)\end{array}$ & 0.86 \\
\hline $\begin{array}{l}\text { ICU admission } \\
\text { Yes } \\
\text { No }\end{array}$ & $\begin{array}{c}17(47.2 \%) \\
5(5.5 \%)\end{array}$ & $\begin{array}{l}15.39 \\
(5.05-46.89)\end{array}$ & $<0.01$ & $\begin{array}{l}45.78 \\
(4.97-420.99)\end{array}$ & $<0.001^{* *}$ \\
\hline $\begin{array}{l}\text { Source of infectio } \\
\text { Bacteremia } \\
\text { Other infection }\end{array}$ & $\begin{array}{r}4(23.5 \%) \\
18(16.4 \%)\end{array}$ & $\begin{array}{l}1.57 \\
(0.46-5.38)\end{array}$ & 0.47 & $\begin{array}{l}1.11 \\
(0.13-9.71)\end{array}$ & 0.73 \\
\hline $\begin{array}{l}\text { Culture } \\
\text { Positive } \\
\text { Negative }\end{array}$ & $\begin{array}{c}19(26.8 \%) \\
3(5.4 \%)\end{array}$ & $\begin{array}{l}6.45 \\
(1.80-23.13)\end{array}$ & 0.004 & $\begin{array}{l}12.71 \\
(1.14-142)\end{array}$ & $0.039^{* *}$ \\
\hline
\end{tabular}


Table 4 Prognostic factors associated with 30-day mortality in febrile neutropenia patients

\begin{tabular}{|c|c|c|c|c|c|}
\hline Variable & $\begin{array}{l}30 \text { day } \\
\text { Mortality Rate }\end{array}$ & $\begin{array}{l}\text { Crude OR } \\
\text { (CI) }\end{array}$ & P-Value & $\begin{array}{l}\text { Adjusted OR } \\
\text { (Cl) }\end{array}$ & P-Value \\
\hline \multicolumn{6}{|l|}{ Age (years) } \\
\hline$\leq 60$ & $16(25.4 \%)$ & 1.36 & 0.5 & 4.13 & 0.21 \\
\hline$>60$ & $10(20 \%)$ & $(0.56-3.33)$ & & $(0.45-33.33)$ & \\
\hline \multicolumn{6}{|l|}{ Gender } \\
\hline Male & $17(24.3 \%)$ & 1.21 & 0.68 & 1.21 & \\
\hline Female & $9(20.9 \%)$ & $(0.49-3.03)$ & & $(0.20-7.25)$ & 0.84 \\
\hline \multicolumn{6}{|l|}{ Race } \\
\hline White & $16(23.2 \%)$ & 0.97 & 0.94 & 0.76 & \\
\hline Hispanic & $9(22 \%)$ & $(0.44-2.16)$ & & $(0.20-3.02)$ & 0.7 \\
\hline African-American & $1(33.3 \%)$ & & & & \\
\hline \multicolumn{6}{|l|}{ Type } \\
\hline $\begin{array}{l}\text { Hematologic Ma- } \\
\text { lignancies }\end{array}$ & $18(24 \%)$ & 1.35 & 0.54 & & \\
\hline Solid tumor & $7(18.9 \%)$ & $(0.51-3.60)$ & & $(0.30-33.15)$ & 0.33 \\
\hline \multicolumn{6}{|l|}{ Comorbidities } \\
\hline Yes & $13(20.8 \%)$ & 1.47 & 0.41 & 1.369 & 0.717 \\
\hline No & $12(27.8 \%)$ & $(0.59-3.66)$ & & $(0.25-7.49)$ & \\
\hline \multicolumn{6}{|l|}{ Fever duration } \\
\hline >4 days & $13(50.0 \%)$ & 5.69 & $<0.001$ & 8.26 & $0.039^{* *}$ \\
\hline$<4$ days & $13(14.9 \%)$ & $(2.16-14.9)$ & & $(1.11-62.50)$ & \\
\hline \multicolumn{6}{|l|}{ Liver enzymes } \\
\hline Abnormal & $9(69.2 \%)$ & 14.82 & $<0.001$ & 48.38 & $<0.001^{\text {** }}$ \\
\hline Normal & $17(17.0 \%)$ & $(3.63-60.52)$ & & $(5.27-444.29)$ & \\
\hline \multicolumn{6}{|l|}{ Albumin level } \\
\hline Low & $7(30.4 \%)$ & 1.64 & 0.35 & 2.26 & 0.38 \\
\hline Normal (> 3g/dL) & $19(21.1 \%)$ & $(0.59-4.54)$ & & $(0.36-14.28)$ & \\
\hline \multicolumn{6}{|l|}{ GFR } \\
\hline$<60$ & $11(32.4 \%)$ & 2.01 & 0.13 & 2.02 & 0.42 \\
\hline$>60$ & $15(19.5 \%)$ & $(0.81-5.01)$ & & $(0.37-11.10)$ & \\
\hline \multicolumn{6}{|l|}{ MASCC } \\
\hline High risk & $22(27.2 \%)$ & 2.61 & 0.1 & 4.649 & 0.15 \\
\hline Low risk & $4(12.5 \%)$ & $(0.82-8.30)$ & & $(0.58-37.53)$ & \\
\hline
\end{tabular}


Table 4 Prognostic factors associated with 30-day mortality in febrile neutropenia patients (Continued)

\begin{tabular}{|c|c|c|c|c|c|}
\hline Variable & $\begin{array}{l}30 \text { day } \\
\text { Mortality Rate }\end{array}$ & $\begin{array}{l}\text { Crude OR } \\
\text { (Cl) }\end{array}$ & P-Value & $\begin{array}{l}\text { Adjusted OR } \\
\text { (CI) }\end{array}$ & P-Value \\
\hline $\begin{array}{l}\text { Neutropenic per } \\
>5 \text { day } \\
\leq 5 \text { day }\end{array}$ & $\begin{array}{c}16(26.7 \%) \\
9(17.6 \%)\end{array}$ & $\begin{array}{l}5.69 \\
(2.61-15.00)\end{array}$ & $<0.001$ & $\begin{array}{l}3.79 \\
(0.60-23.81)\end{array}$ & 0.157 \\
\hline $\begin{array}{l}\text { ICU admission } \\
\text { yes } \\
\text { no }\end{array}$ & $\begin{array}{c}18(56.2 \%) \\
8(9.9 \%)\end{array}$ & $\begin{array}{l}11.73 \\
(4.272-32.22)\end{array}$ & $<0.001$ & \begin{tabular}{|l}
63.66 \\
$(5.96-680.30)$
\end{tabular} & $<0.001^{* *}$ \\
\hline $\begin{array}{l}\text { Source of infect } \\
\text { Bacteremia } \\
\text { Other infection }\end{array}$ & $\begin{array}{r}4(26.7 \%) \\
22(22.4 \%)\end{array}$ & \begin{tabular}{|l}
1.26 \\
$(0.36-4.34)$
\end{tabular} & 0.72 & $\begin{array}{l}1.52 \\
(0.14-16.22)\end{array}$ & 0.73 \\
\hline $\begin{array}{l}\text { Culture } \\
\text { Positive } \\
\text { Neqative }\end{array}$ & $\begin{array}{c}20(31.7 \%) \\
6(12 \%)\end{array}$ & $\begin{array}{l}3.41 \\
(1.25-9.31)\end{array}$ & 0.017 & $\begin{array}{l}3.32 \\
(0.48-23.11)\end{array}$ & 0.23 \\
\hline
\end{tabular}

Table 5 Prognostic factor associated with more than 8 days length of stay (LOS) in febrile neutropenia patients

\begin{tabular}{|c|c|c|c|c|c|}
\hline Variable & LOS & $\begin{array}{l}\text { Crude OR } \\
(\mathrm{Cl})\end{array}$ & P-Value & $\begin{array}{l}\text { Adjusted OR } \\
\text { (Cl) }\end{array}$ & P-Value \\
\hline \multicolumn{6}{|l|}{ Age (years) } \\
\hline$\leq 60$ & $34(50 \%)$ & 1.68 & 0.5 & 2.84 & 0.07 \\
\hline$>60$ & $22(37.3 \%)$ & $(0.83-3.42)$ & & $(0.90-8.93)$ & \\
\hline \multicolumn{6}{|l|}{ Gender } \\
\hline Male & $35(43.8 \%)$ & 1.04 & 0.92 & 1.2 & 0.74 \\
\hline Female & $21(44.7 \%)$ & $(0.50-2.14)$ & & $(0.41-3.53)$ & \\
\hline \multicolumn{6}{|l|}{ Race } \\
\hline White & $38(46.9 \%)$ & 0.83 & 0.59 & 0.87 & 0.76 \\
\hline Hispanic & $16(37.2 \%)$ & $(0.43-1.62)$ & & $(0.36-2.14)$ & \\
\hline African-American & $2(66.7 \%)$ & & & & \\
\hline \multicolumn{6}{|l|}{ Type } \\
\hline Hematologic Malignancy & $48(57.1 \%)$ & 5.67 & $<0.001$ & 4.06 & $0.013^{\star *}$ \\
\hline Solid tumor & & $(2.34-13.70)$ & & $(1.34-12.31)$ & \\
\hline & $8(19.1 \%)$ & & & & \\
\hline \multicolumn{6}{|l|}{ Comorbidities } \\
\hline Yes & $34(40 \%)$ & 0.6 & 0.188 & 0.59 & 0.33 \\
\hline No & 22(52.4\%) & $(0.29-1.28)$ & & $(0.21-1.69)$ & \\
\hline
\end{tabular}


Table 5 Prognostic factor associated with more than 8 days length of stay (LOS) in febrile neutropenia patients (Continued)

\begin{tabular}{|c|c|c|c|c|c|}
\hline Variable & LOS & $\begin{array}{l}\text { Crude OR } \\
\text { (CI) }\end{array}$ & P-Value & $\begin{array}{l}\text { Adjusted OR } \\
\text { (Cl) }\end{array}$ & P-Value \\
\hline $\begin{array}{l}\text { Fever duration } \\
>4 \text { days } \\
<4 \text { days }\end{array}$ & $\begin{array}{l}24(77.4 \%) \\
32(33.3 \%)\end{array}$ & $\begin{array}{l}6.86 \\
(2.67-17.60)\end{array}$ & $<0.001$ & $\begin{array}{l}6.29 \\
(1.81-21.92)\end{array}$ & $0.004^{* *}$ \\
\hline $\begin{array}{l}\text { Liver enzymes } \\
\text { Abnormal } \\
\text { Normal }\end{array}$ & $\begin{array}{r}6(46.2 \%) \\
50(43.9 \%)\end{array}$ & $\begin{array}{l}1.097 \\
(0.35-3.47)\end{array}$ & 0.88 & $\begin{array}{l}1.97 \\
(0.43-8.93)\end{array}$ & 0.38 \\
\hline $\begin{array}{l}\text { Albumin level } \\
\text { Low } \\
\text { Normal (> } 3 \mathrm{~g} / \mathrm{dL})\end{array}$ & $\begin{array}{l}15(51.7 \%) \\
41(41.8 \%)\end{array}$ & $\begin{array}{l}1.49 \\
(0.65-3.42)\end{array}$ & 0.35 & $\begin{array}{l}1.15 \\
(0.35-3.72)\end{array}$ & 0.82 \\
\hline $\begin{array}{l}\text { GFR } \\
<60 \\
>60\end{array}$ & $\begin{array}{l}18(48.6 \%) \\
38(42.7 \%)\end{array}$ & $\begin{array}{l}1.27 \\
(0.59-2.74)\end{array}$ & 0.54 & $\begin{array}{l}2.09 \\
(0.72-6.05)\end{array}$ & 0.17 \\
\hline $\begin{array}{l}\text { MASCC } \\
\text { High risk } \\
\text { Low risk }\end{array}$ & $\begin{array}{l}44(47.8 \%) \\
15(34.3 \%)\end{array}$ & $\begin{array}{l}1.75 \\
(0.78-3.95)\end{array}$ & 0.1 & $\begin{array}{l}2.32 \\
(0.67-8.03)\end{array}$ & 0.19 \\
\hline $\begin{array}{l}\text { Neutropenic period } \\
>5 \text { day } \\
<5 \text { day }\end{array}$ & $\begin{array}{l}42(60.9 \%) \\
14(25 \%)\end{array}$ & $\begin{array}{l}4.67 \\
(2.15-10.21)\end{array}$ & $<0.001$ & $\begin{array}{l}3.68 \\
(1.44-9.40)\end{array}$ & $0.007^{* *}$ \\
\hline $\begin{array}{l}\text { ICU admission } \\
\text { yes } \\
\text { no }\end{array}$ & $\begin{array}{l}20(55.6 \%) \\
36(39.6 \%)\end{array}$ & $\begin{array}{l}1.91 \\
(0.88-4.17)\end{array}$ & 0.1 & $\begin{array}{l}1.29 \\
(0.41-4.13)\end{array}$ & 0.78 \\
\hline $\begin{array}{l}\text { Source of infection } \\
\text { Bacteremia } \\
\text { Other infection }\end{array}$ & $\begin{array}{r}7(41.2 \%) \\
49(44.5 \%)\end{array}$ & $\begin{array}{l}0.87 \\
(0.31-2.46)\end{array}$ & 0.87 & $\begin{array}{l}0.62 \\
(0.14-2.78)\end{array}$ & 0.53 \\
\hline $\begin{array}{l}\text { Culture } \\
\text { Positive } \\
\text { Negative }\end{array}$ & $\begin{array}{l}36(52.7 \%) \\
20(35.7 \%)\end{array}$ & $\begin{array}{l}1.85 \\
(0.90-3.80)\end{array}$ & 0.09 & $\begin{array}{l}1.67 \\
(0.53-5.24)\end{array}$ & 0.38 \\
\hline
\end{tabular}

ity, especially in the non-Hodgkin lymphoma (NHL) group. Besides hematologic malignancy, several factors contributed to prolonged LOS and increased risk of death, including older age, impaired kidney function, and severity of neutropenia. Bacteremia and pneumonia significantly increased the mortality. 2, 4-8 Our study showed similar results in factors that af- fect LOS, which were neutropenic duration and type of hematologic malignancy. Impaired kidney function could occur with sepsis, dehydration, or chronic kidney disease, but did not show any correlation with outcome in our cohort. Severe clinical burden (e.g., ICU admission) showed a significant correlation with mortality rate. 
Invasive fungal infection also contributed to an increased risk of death in some studies. ${ }^{2,7}$ However, in our study there was only one culture proven fungal infection which prevented more analysis. Fever duration was associated with a 30-day mortality rate in our sample, which could reflect inadequate coverage of antibiotics to ongoing infection, severe underlying hematologic malignancy, or an immunosuppressed state.

C-reactive protein (CRP) seems to predict adverse outcomes. ${ }^{8-10} \mathrm{CRP}$ was not routinely obtained in patients admitted for infection in our hospital; therefore, we did not have enough data to analyze this particular test. Recent studies have shown hematologic parameters could be used as an inflammatory marker. Red blood cell distribution width (RDW) was reported as a predictor of mortality rate among patients with critical illness and infectious disease, such as community acquired pneumonia, sepsis, and septic shock. ${ }^{11-15}$ Thrombocytopenia has been associated with poor outcomes in febrile neutropenia. ${ }^{5,8,16}$ Correlation between sepsis from febrile neutropenia and RDW has never been explored. Our study collected platelet counts and RDW values at the onset of fever and at 24,48 , and 72 hours to assess any relation to mortality rate, but the results were not significant (data not shown). There were two possible explanations for these events. First, RDW and platelet levels are dynamic variables with changes associated with acute disease states. In cancer patients, who have ongoing illness, this response might not occur in patients with chronic disease. The second explanation could be that our study population had received chemotherapy agents which could cause an inadequate bone marrow response and therefore prevent significant change in hematologic parameters.

Our study found significant correlation between abnormal liver enzymes (aspartate aminotransferase, AST; alanine aminotransferase, ALT, and alkaline phosphatase, AKLP) with both in-patient and 30-day mortality rates. These markers had not been used in earlier studies. Low total protein levels do predict outcomes. ${ }^{8}$ We suggest that these abnormal liver enzymes developed secondary to liver involvement from severe sepsis and that this could lead to a higher mortality rate in this group of patients.
The MASCC risk index has been used widely to identify low risk febrile neutropenia. Multiple studies have shown that the tool has a sensitivity range from 59 to $95 \% .{ }^{17}$ Positive predictive values of MASCC score in those studies were $85 \%$ or higher when study populations presented with mostly solid tumors. The MASCC might not be accurate in predicting outcomes in hematologic malignancy. As seen in our study with a majority of patients with hematologic malignancy $(66.1 \%)$, mortality did not show a significant correlation with this risk index. Nevertheless, a score less than 21 has been associated with poor outcomes in febrile neutropenia. ${ }^{8,10,16,18}$ Several studies have shown that MASCC could predict an unfavorable outcomes in febrile neutropenia when combined with other inflammatory markers, such as procalcitonin or $\mathrm{CRP},{ }^{10},{ }^{19}$ when compared to inflammatory markers alone. ${ }^{20}$ MASCC is still currently the best risk stratification tool for febrile neutropenia, ${ }^{21}$ but it does not include neutropenic duration, ${ }^{17}$ which was an independent risk factor for prolonged LOS in our study.

In summary, our study explored clinical risk factors associated with outcomes in febrile neutropenia in chemotherapy patients. Our results were similar to prior studies. The use of MASCC should aid physicians in identifying high risk patients and prompt immediate treatment. Clinical risk factors, such as the type of underlying malignancy and fever and neutropenic duration, should be considered when evaluating patients with febrile neutropenia.

Author Affiliation: Drs. Nattamol Hosiriluck, Saranapoom
Klomjit, and Supannee Rassameehiran are residents in
the Department of Internal Medicine at TTUHSC. Dr. Grerk
Sutamtewagul is a fellow in hematology and oncology at
the University of lowa in lowa City, IA. Drs. Lukman Tijani
and Saba Radhi are faculty members in the Division of
Hematology and Oncology at TTUHSC.

Received: $11 / 25 / 2014$

Accepted: 1/08/2015

Reviewers: Cynthia Jumper MD

Published electronically: 01/15/2015

Conflict of Interest Disclosures: None 


\section{REFERENCES}

1. Schuette HL, Tucker TC, Brown ML, Potosky AL, Samuel T. The costs of cancer care in the United States: implications for action. Oncology (Williston Park) 1995; 9(11 Suppl):19-22.

2. Kuderer NM, Dale DC, Crawford J, Cosler LE, Lyman GH. Mortality, morbidity, and cost associated with febrile neutropenia in adult cancer patients. Cancer 2006; 106(10):2258-66.

3. Klastersky J, Paesmans M, Rubenstein EB, Boyer M, Elting L, Feld R, et al. The Multinational Association for Supportive Care in Cancer risk index: A multinational scoring system for identifying low-risk febrile neutropenic cancer patients. J Clin Oncol 2000; 18(16):3038-51.

4. Lal A, Bhurgri Y, Rizvi N, Virwani M, Memon RU, Saeed W, et al. Factors influencing in-hospital length of stay and mortality in cancer patients suffering from febrile neutropenia. Asian Pac J Cancer Prev 2008; 9(2):303-8.

5. Lee YM, Lockwood C. Prognostic factors for risk stratification of adult cancer patients with chemotherapyinduced febrile neutropenia: a systematic review and metaanalysis. Int J Nurs Pract 2013;19 (6):557-76.

6. Lyman GH, Michels SL, Reynolds MW, Barron R, Tomic KS, Yu J. Risk of mortality in patients with cancer who experience febrile neutropenia. Cancer 2010; 116(23):555563.

7. Chindaprasirt J, Wanitpongpun C, Limpawattana P, Thepsuthammarat K, Sripakdee W, Sookprasert A, et al. Mortality, length of stay, and cost associated with hospitalized adult cancer patients with febrile neutropenia. Asian Pac J Cancer Prev 2013; 14(2):1115-9.

8. Gunalp M, Koyunoglu M, Gurler S, Koca A, Yesilkaya I, Oner E, et al. Independent factors for prediction of poor outcomes in patients with febrile neutropenia. Med Sci Monit 2014; 20:1826-32.

9. Povoa P, Souza-Dantas VC, Soares M, Salluh JF. C-reactive protein in critically ill cancer patients with sepsis: influence of neutropenia. Crit Care 2011; 15(3):R129.

10. Combariza JF, Lombana M, Pino LE, Arango M. C-reactive protein and the MASCC risk index identify highrisk patients with febrile neutropenia and hematologic neoplasms. Support Care Cancer. 2014.

11. Kim CH, Park JT, Kim EJ, Han JH, Han JS, Choi JY, et al. An increase in red blood cell distribution width from baseline predicts mortality in patients with severe sepsis or septic shock. Crit Care 2013; 17(6):R282.

12. Braun E, Domany E, Kenig Y, Mazor Y, Makhoul BF, Azzam ZS. Elevated red cell distribution width predicts poor outcome in young patients with community acquired pneumonia. Crit Care 2011; 15(4):R194.
13. Ku NS, Kim HW, Oh HJ, Kim YC, Kim MH, Song $\mathrm{JE}$, et al. Red blood cell distribution width is an independent predictor of mortality in patients with gram-negative bacteremia. Shock 2012; 38(2):123-7.

14. Lee JH, Chung HJ, Kim K, Jo YH, Rhee JE, Kim $\mathrm{YJ}$, et al. Red cell distribution width as a prognostic marker in patients with community-acquired pneumonia. Am J Emerg Med 2013; 31(1):72-9.

15. Jo YH, Kim K, Lee JH, Kang C, Kim T, Park HM, et al. Red cell distribution width is a prognostic factor in severe sepsis and septic shock. Am J Emerg Med 2013; 31(3):545-8.

16. Ahn S, Lee YS, Lim KS, Lee JL. Adding procalcitonin to the MASCC risk-index score could improve risk stratification of patients with febrile neutropenia. Support Care Cancer 2013 21(8):2303-8.

17. Klastersky J, Paesmans M. The Multinational Association for Supportive Care in Cancer (MASCC) risk index score: 10 years of use for identifying low-risk febrile neutropenic cancer patients. Support Care Cancer 2013; 21(5):1487-95.

18. Shaw AT, Kim DW, Nakagawa K, Seto T, Crino L, Ahn MJ, et al. Crizotinib versus chemotherapy in advanced ALK-positive lung cancer. N Engl J Med 2013; 368(25):2385-94.

19. Ahn S, Lee YS. Predictive factors for poor prognosis febrile neutropenia. Curr Opin Oncol 2012;24(4):37680.

20. Uys A, Rapoport BL, Fickl H, Meyer PW, Anderson R. Prediction of outcome in cancer patients with febrile neutropenia: comparison of the Multinational Association of Supportive Care in Cancer risk-index score with procalcitonin, C-reactive protein, serum amyloid A, and interleukins-1beta, -6, -8 and -10. Eur J Cancer Care (Engl) 2007; 16(6):475-83.

21. Hui EP, Leung LK, Poon TC, Mo F, Chan VT, Ma AT, et al. Prediction of outcome in cancer patients with febrile neutropenia: a prospective validation of the Multinational Association for Supportive Care in Cancer risk index in a Chinese population and comparison with the Talcott model and artificial neural network. Support Care Cancer 2011; 19(10):1625-35. 